$\underline{\text { Stereotype threat as a trigger of mind-wandering in older adults }}$

By: Megan L. Jordano and Dayna R. Touron ${ }^{*}$

Jordano, M. L. \& Touron, D. R. (2017). Stereotype threat as a trigger of mind wandering in older adults. Psychology and Aging, 32(3), 37-313.

(C)American Psychological Association, 2017. This paper is not the copy of record and may not exactly replicate the authoritative document published in the APA journal. Please do not copy or cite without author's permission. The final article is available, upon publication, at: http://dx.doi.org/10.1037/pag0000167 ; http://dx.doi.org/10.1037/pag0000167.supp (Supplemental)

\begin{abstract}
:
Older adults (OAs) report less overall mind-wandering than younger adults (YAs) but more taskrelated interference (TRI; mind-wandering about the task). The current study examined TRI while manipulating older adults' performance-related concerns. We compared groups for which memory-related stereotype threat (ST) was activated or relieved to a control group. Participants completed an operation span task containing mind-wandering probes. ST-activated OAs reported more TRI than ST-relieved OAs and had worse performance on the operation span task. This study illustrates that environmental context triggers current concerns and determines, in part, the frequency and content of mind-wandering.
\end{abstract}

Keywords: mind-wandering | stereotype threat | executive control

\title{
Article:
}

\footnotetext{
* Touron, Dayna R., Department of Psychology, The University of North Carolina at Greensboro, 296 Eberhart Building, P.O. Box 26170, Greensboro, NC, US, 27402-6170,d_touron@uncg.edu
} 
Mind-wandering is a common experience. Younger adults (YAs) report mind-wandering about things unrelated to the task (task-unrelated thoughts; TUTs) $30 \%-50 \%$ of the time in everyday life (Kane et al., 2007; Killingsworth \& Gilbert, 2010) and 50-70\% of the time during laboratory tasks (Jackson \& Balota, 2012; McVay, Meier, Touron, \& Kane, 2013). Various factors influence mind-wandering. For example, YAs with lower executive control abilities mindwander more than YAs with higher executive control on challenging tasks (McVay \& Kane, 2009; Mrazek, Franklin, Phillips, Baird, \& Schooler, 2013; Rummel \& Boywitt, 2014; Unsworth \& McMillan, 2013). It is not surprising that, in-the-moment TUTs are also associated with impaired cognitive performance (Smallwood, McSpadden, \& Schooler, 2008; McVay \& Kane, 2012a). In addition to TUTs, individuals may mind-wander about the task itself (task-related interference; TRI). For example, one might monitor their task performance or mind-wander about task strategies. Although TRI experiences are tangentially related to the task, they are not directly about responding to task stimuli. Furthermore, both TUTs and TRI (McVay \& Kane, 2012b) are associated with errors on cognitive tasks. Therefore, TRI is characterized as mindwandering. The mind-wandering literature has principally focused on TUTs rather than TRI, and earlier mind-wandering studies did not distinguish TRI, so participants may have misclassified TRI experiences either as on-task or as TUTs.

\section{MIND-WANDERING AND AGING}

Normal aging is characterized by cognitive changes, with performance declining on numerous abilities, including executive control (Hasher \& Zacks, 1988; Salthouse, 1991; Schaie, 1994). It has been proposed that executive control deficits allow off-task thoughts to enter consciousness (McVay \& Kane, 2010). Because OAs have worse executive control capabilities than do YAs, one might expect OAs to mind-wander more than YAs. It is surprising that the opposite is found, with OAs reporting fewer TUTs and less overall mind-wandering than YAs (Frank, Nara, Zavagnin, Touron, \& Kane, 2015; Giambra, 1989; Grodsky \& Giambra, 1990; Jackson \& Balota, 2012; Krawietz, Tamplin, \& Radvansky, 2012). However, OAs report more TRI than do YAs (Frank et al., 2015; Jackson \& Balota, 2012; Krawietz et al., 2012; McVay et al., 2012a, 2013). This can be explained by a theory that proposes that mind-wandering occurs when one's current concerns trigger off-task thoughts, and executive control fails to inhibit them (McVay \& Kane, 2010). OAs might TUT less and experience TRI more than YAs in laboratory settings because the context triggers different concerns. Being on a familiar college campus may trigger everyday concerns in YAs, increasing TUTs. That same testing environment may trigger concerns about cognitive decline in OAs (Hertzog \& Hultsch, 2000), increasing performance monitoring and thus TRI. The current study directly tests the "Control Failures $\times$ Current Concerns" $(\mathrm{CF} \times \mathrm{C})$ framework (McVay \& Kane, 2010) by manipulating performance-related concerns in OAs.

\section{MIND-WANDERING, AGING, AND STEREOTYPE THREAT}

Stereotype threat (ST) is defined as being at risk for behaving in a way that confirms a negative stereotype about a group one belongs to (Steele \& Aronson, 1995). Many individuals believe that aging particularly leads to memory impairment (Hummert, 1999). Although structural changes in the brain are one cause of memory deficits in OAs, social factors may also contribute. For example, when OAs are exposed to negative stereotypes about aging and cognition, their memory performance suffers (for a review, see Barber \& Mather, 2014). Factors such as 
increased physiological stress, effortful suppression of negative emotions, and performance monitoring have been proposed as mechanisms by which ST leads to performance deficits (Schmader, Johns, \& Forbes, 2008). It has been suggested that, if one is using their cognitive resources to suppress negative emotions and to engage in metacognitions, then fewer resources are left to respond optimally to task demands (Schmader, Forbes, Zhang, \& Mendes, 2009; Schmader et al., 2008; Schuster, Martiny, \& Schmader, 2015).

Although past research shows that priming of performance-related concerns increases worryladen task evaluation (Beilock, Rydell, \& McConnell, 2007; Cadinu, Maass, Rosabianca, \& Kiesner, 2005) and it has been proposed that ST increases TRI, research in the ST literature typically measures off-task thoughts using retrospective questions that may not ask about both TUTs and TRI (Cadinu et al., 2005). Retrospective questionnaires also require participants to report their mind-wandering experiences after testing has finished, potentially resulting in participants overlooking or forgetting some mind-wandering episodes (Beilock et al., 2007; Smallwood et al., 2008).

Studies have examined the effect of ST on probe-caught mind-wandering. Mrazek et al. (2011) examined the effect of math-gender ST in female YAs. Thought probes within a math task asked participants to indicate, on a Likert-scale from 1 to 10, the degree to which they mindwandered before the probe appeared. A retrospective measure of TUTs and TRI was also administered after the task. It was found that ST led to worse math performance, and a simultaneous regression predicting probe-caught mind-wandering from retrospective TUTs and TRI revealed that the thought probes were more strongly related to retrospective TUTs rather than retrospective TRI. However, there may have been methodological limitations. The online probes did not include different mind-wandering categories to choose from, so the results were dependent on participants being able to accurately remember what they mind-wandered about during testing. In our lab, we have completed two studies examining the effect of activating math-gender ST on math performance in female YAs (Jordano \& Touron, 2015). In both studies, ST activation increased probe-caught TRI. These YA studies suggest that the environmental cuing of performance concerns influences the frequency and content of mind-wandering, lending support for the “CF × C” framework (McVay \& Kane, 2010).

\section{CURRENT AIMS}

The current study further tested the CF × C framework (McVay \& Kane, 2010) by systematically activating and relieving memory-related ST in OAs. Other work has supported this framework by priming everyday personal concerns in YAs and finding increased TUTs but not TRI (Kopp, D’Mello, \& Mills, 2015; Masicampo \& Baumeister, 2011; McVay \& Kane, 2013). However, the current study is the first to activate performance-related concerns in OAs to determine potential causes of OAs' activated TRI. Although there has been concern that OAs thought reports may be less valid than those of YAs due to OAs either having an unawareness of their mind-wandering experiences or unwillingness to report mind-wandering, eye-tracking data indicate that OAs' thought reports are as valid as YAs' thought reports (Frank et al., 2015). We predicted that OAs with activated performance-related concerns would report more TRI and would have worse memory performance than OAs relieved of performance-related concerns. 


\section{METHOD}

\section{Participants}

We tested 30 YAs (18-25, Mage $=19.20$ years, SDage $=1.16$ years) and 90 OAs $(60-75$, Mage $=67.51$ years, SDage $=3.89$ years). This study was approved by the Institutional Review Board at The University of North Carolina at Greensboro. YAs were undergraduates participating for course credit. OAs were community volunteers paid a modest honorarium. All participants scored greater than 20/50 on a near visual acuity test and none had a history of stroke or dementia. OAs in the control $(M=.2 .148, S E=.237)$ and ST relief $(M=2.067, S E=.377)$ groups reported taking more medications than YAs $(M=.867, S E=.184 ; p s<.05)$. OAs in the ST activated group $(M=1.704, S E=.324)$ reported taking more medications than YAs, although this difference was not significant $(p>.05)$. OAs in the control $(M=33.400, S E=1.556)$, ST relief $(M=31.300, S E=1.196)$, and ST activated $(M=30.533, S E=1.176)$ groups scored lower on processing speed (Pattern Comparison; Salthouse, 1993) than YAs $(M=35.833, S E=$ 1.337; $p s<.05)$. Finally, OAs in the control $(M=23.367, S E=1.177)$, ST relief $(M=$ 24.200, $S E=1.222)$, and ST activated $(M=23.033, S E=1.253)$ groups had higher vocabulary (Ekstrom, French, \& Harman, 1976) than YAs $(M=13.733, S E=.663$; $p s<.05)$. Importantly, there were no differences between OA groups in age, medications, processing speed, and vocabulary ( $p s>.05)$.

\section{Materials and Procedure}

\section{Stereotype threat manipulation}

OAs were randomly assigned to one of three conditions: relief of memory-related ST, activation of memory-related ST, and control. Thirty OAs underwent a manipulation to activate ST, which involved reading two fictitious newspaper articles (Hess, Auman, Colcombe, \& Rahhal, 2003) that characterized age-related memory changes as biologically driven and inevitable, and were told that they would soon complete a memory task. Thirty OAs relieved of ST read two fictitious newspaper articles that described age-related memory changes as mediated by lifestyle practices and were told that they would soon complete a math task. To avoid demand characteristics, participants were told the article was for other research and rated article comprehensibility. Thirty OA and 30 YA control participants received no ST intervention. Experimental task instructions can be found in the Table S1 in the online supplemental materials.

\section{Operation span task (OSPAN)}

After completing a health and demographics questionnaire and the measures of visual acuity, processing speed, and vocabulary, participants completed the automated OSPAN task (Conway et al., 2005). The OSPAN is a working memory task that requires participants to alternate between verifying simple math equations and studying letters for a later recall task. At the end of each trial, participants must recall, in correct serial order, the letters presented during the trial. The OSPAN used consisted of 81 trials, with 27 trials each of set sizes (numbers of consecutive equation/letter pairs) of 3,4 , and 5 . We chose the OSPAN because it could be framed as either a math task or as a memory task, depending on the condition. During the OSPAN participants 
responded to 9 thought probes which appeared at quasi-random intervals approximately every 2 min (see Seli, Carriere, Levene, \& Smilek, 2013). Thought probes could appear (a) after participants saw the final math equation within a trial, but before they verified the answer for that equation, (b) after the final math equation within the trial had been verified, but before the final letter to be remembered was presented, or (c) at the end of the trial after the participant saw the last letter to be remembered. Participants were instructed to indicate what they were thinking about immediately before the probe appeared, and were provided with descriptions of the response choices: (a) the task; (b) task approach; (c) task evaluation, (d) everyday things, (e) current state of being, (f) personal worries, (g) daydreams, or (h) other.

Using the probe responses, we calculated mean proportions of TUT, TRI, and on-task thoughts. We separated TRI into two subtypes. It has been suggested that ST primarily increases worryladen monitoring of task performance, which we label "reactive TRI," corresponding to Option 3 above. However, it is possible that TRI can also be about task approach or strategy, which we label "proactive TRI," corresponding to Option 2 above. In two previous experiments looking at math-gender ST in female YAs, control and ST participants reported both TRI subtypes, with ST participants reporting more overall TRI, proactive TRI, and reactive TRI.

Prior to completing the OSPAN, participants were told that mind-wandering within the lab is normal and expected, to de-stigmatize mind-wandering and encourage honest reporting. Participants practiced the math verification portion, the letter recall portion, and the math verification and letter recall portions together before data collection began. Math verification and letter recall accuracies were recorded during the OSPAN. During the task, participants received feedback regarding trial-level letter recall accuracy, trial level math verification accuracy, and cumulative math verification accuracy. Participants were asked to try to keep their cumulative math verification accuracy at least $85 \%$ correct. The difficulty of the math verifications problems was held constant across participants and the amount of time participants had to complete the math verification trials was determined by their average trial completion time during practice.

\section{Additional measures}

A variety of computerized posttask questionnaires were administered. The Dundee Stress State Questionnaire is a retrospective measure of thought content (DSSQ; Matthews, Joyner, Gilliland, Huggins, \& Falconer, 1999) that includes eight questions assessing TUTs (e.g., "I thought about something that happened to me earlier") and eight questions assessing TRI (e.g., "I thought about how I should work more carefully"). The DSSQ has been used in prior work and was included to determine if retrospective reports of mind-wandering were similar to probe-caught reports of mind-wandering.

An additional goal of the current study was to examine factors that influence mind-wandering and performance. We conducted exploratory mediational analyses to gain a better understanding of factors expected to influence amount of TRI and OSPAN performance; these can be found in the online supplemental materials. We included posttask questions about self-rated task difficulty, focus, stress, and effort, and an affect scale (Positive and Negative Affect Schedule [PANAS]; Watson, Clark, \& Tellegen, 1988; Kane et al., 2007; Smallwood, Fitzgerald, Miles, \& Phillips, 2009). Because ST effects may be mediated by importance of good performance in the 
stereotype domain (Ennis, Hess, \& Smith, 2013) and anxiety during completion of the stereotyped task (Chasteen, Bhattacharyya, Horhota, Tam, \& Hasher, 2005), we included the Anxiety and Achievement subscales of the Metamemory in Adulthood Questionnaire (MIA; Dixon \& Hultsch, 1984). The means and standard errors for these measures are listed in Table 1. Prior to debriefing, participants were asked to write down what they believed the study was about. No participants indicated that they believed the study was about age-related ST in mindwandering experiences.

Table 1

Descriptive Statistics (Means and Standard Errors) for Each Group

\begin{tabular}{|c|c|c|c|c|c|c|c|c|}
\hline \multirow[b]{2}{*}{ Variable } & \multicolumn{2}{|c|}{ YA } & \multicolumn{2}{|c|}{$\mathrm{OA}$ control } & \multicolumn{2}{|c|}{ OA relief } & \multicolumn{2}{|c|}{ OA activated } \\
\hline & $M$ & $S E$ & $M$ & $S E$ & $M$ & $S E$ & $M$ & $S E$ \\
\hline Age & 19.20 & 0.21 & 66.52 & 0.69 & 67.90 & 0.69 & 68.11 & 0.86 \\
\hline Education & 12.80 & 0.22 & 16.67 & 0.33 & 16.07 & 0.53 & 15.60 & 0.26 \\
\hline Vocabulary & 13.73 & 0.66 & 22.93 & 1.22 & 24.20 & 1.22 & 23.03 & 1.25 \\
\hline Processing speed & 35.83 & 1.34 & 33.23 & 1.58 & 31.33 & 1.20 & 30.77 & 1.19 \\
\hline Medications & 0.87 & 0.18 & 2.03 & 0.24 & 2.07 & 0.38 & 1.63 & 0.30 \\
\hline OT & 0.62 & 0.05 & 0.68 & 0.06 & 0.73 & 0.05 & 0.57 & 0.07 \\
\hline TUT & 0.26 & 0.05 & 0.08 & 0.04 & 0.09 & 0.03 & 0.07 & 0.04 \\
\hline TRI & 0.12 & 0.03 & 0.25 & 0.05 & 0.18 & 0.04 & 0.35 & 0.06 \\
\hline Proactive TRI & 0.06 & 0.02 & 0.11 & 0.02 & 0.08 & 0.02 & 0.17 & 0.04 \\
\hline Reactive TRI & 0.06 & 0.01 & 0.15 & 0.04 & 0.10 & 0.03 & 0.18 & 0.04 \\
\hline Recall accuracy & 87.22 & 1.64 & 83.86 & 3.01 & 86.86 & 2.34 & 76.74 & 3.56 \\
\hline DSSQ TUT & 13.07 & 0.99 & 9.79 & 0.61 & 9.38 & 0.59 & 9.90 & 0.57 \\
\hline DSSQ TRI & 20.27 & 1.17 & 18.10 & 0.94 & 18.28 & 0.83 & 20.52 & 0.99 \\
\hline Recall difficulty & 2.80 & 0.19 & 2.93 & 0.23 & 2.62 & 0.90 & 3.14 & 0.23 \\
\hline Overall difficulty & 2.37 & 0.19 & 2.76 & 0.18 & 2.62 & 0.21 & 3.00 & 0.93 \\
\hline Recall focus & 3.93 & 0.19 & 4.52 & 0.14 & 4.52 & 0.18 & 4.34 & 0.21 \\
\hline Stress & 2.73 & 0.23 & 2.28 & 0.24 & 2.69 & 0.21 & 2.48 & 0.20 \\
\hline Fatigue & 3.70 & 0.19 & 2.79 & 0.22 & 2.66 & 0.19 & 3.10 & 0.22 \\
\hline Effort & 3.80 & 0.21 & 4.31 & 0.20 & 4.45 & 0.15 & 4.28 & 0.20 \\
\hline Stereotype awareness & 3.33 & 0.22 & 3.79 & 0.17 & 4.10 & 0.20 & 4.34 & 0.17 \\
\hline MIA achievement & 53.67 & 1.50 & 52.55 & 0.98 & 53.62 & 0.95 & 53.41 & 0.95 \\
\hline MIA anxiety & 40.77 & 1.48 & 35.45 & 1.64 & 35.07 & 1.07 & 38.93 & 1.48 \\
\hline Positive mood & 26.57 & 1.65 & 35.31 & 1.22 & 36.55 & 1.34 & 34.59 & 0.91 \\
\hline Negative mood & 15.77 & 0.58 & 14.03 & .84 & 13.48 & 1.03 & 13.31 & 0.71 \\
\hline
\end{tabular}

Note. $\quad \mathrm{YA}=$ younger adults: $\mathrm{OA}=$ Older adults: $\mathrm{Age}=$ Chronological age in years; Education $=$ years of education; Vocabulary $=$ score on Advanced Vocabulary Test (Ekstrom et al., 1976); Processing speed = number correct on the Pattern Comparison Test; Medications = number of daily medications; OT = proportion of probe-caught on-task thoughts; TUT = proportion of probe-caught task-unrelated thoughts; TRI = proportion of probe-caught task-related interference; Proactive TRI = proportion of probe-caught TRI regarding task approach or strategy; Reactive TRI = proportion of probe-caught TRI regarding evaluation of task performance; Recall accuracy $=$ accuracy on the letter recall portion of the operation span task (OSPAN); DSSQ TUT $=$ score on the TUT subscale of the Dundee Stress State Questionnaire (DSSQ; out of 40), with higher scores indicating more TUTs; DSSQ TRI = score on the TRI subscale of the DSSQ (out of 40), with higher scores indicating more TRI; Overall difficulty = self-rated perceived difficulty of the OSPAN task overall (out of 5 , where $1=$ not at all difficult, $5=$ very difficult; Recall focus = self-rated focus on the letter recall portion of the OSPAN (out of 5 , where $1=$ not at all focused, $5=$ very much focused $)$; Stress = self-rated perceived stress or anxiety during the OSPAN (out of 5 , where $1=$ not at all stressed, $5=$ very stressed); Fatigue $=$ how fatiguing the OSPAN was perceived to be (out of 5 , where $1=$ not at all fatiguing, $5=$ very fatiguing); Effort $=$ self-rated effort during the OSPAN task (out of 5 , where $1=$ no effort at all, $5=a$ lot of effort); Stereotype awareness $=$ self-rated belief that a negative stereotype regarding aging and cognitive decline exists (out of 5, where $1=$ strongly disagree that there is a stereotype that OAs are inferior to YAs in terms of cognitive ability, $5=$ strongly agree that there is a stereotype that OAs are inferior to YAs in terms of cognitive ability): MIA Achievement $=$ Score on the memory achievement scale of the Metamemory in Adulthood Questionnaire (MIA; out of 75), with higher scores indicating more self-rated importance of good memory performance; MIA Anxiety = Score on the memory anxiety scale of the MIA (out of 65), with higher scores indicating more self-rated anxiety while completing daily task that have a memory component; Positive mood $=$ score on the positive affect scale of the Positive and Negative Affect Schedule (PANAS; out of 50), with higher scores indicating more positive mood; Negative mood = score on the negative affect scale of the PANAS (out of 50), with higher scores indicating more negative mood.

\section{RESULTS}

\section{TUTs, TRI, and OSPAN Performance}

We predicted that the YA group would report more TUTs than all OA groups. As expected, there was an effect of condition on TUTs, $F(3,116)=4.856, p=.003, \eta^{2}=.112$, with YAs reporting a higher proportion of TUTs than control OAs $(d=.726)$, ST relief OAs $(d=.753)$, and ST 
activated OAs ( $d=.766 ; p$ s $<.05$; see Table 1 and Figure 1$)$. There were no differences between control and ST relief OAs $(d=.052)$, control and ST activated OAs $(d=.046)$, and ST activated and relief OAs $(d=.103 ; p s>.05)$. Most critically, we also predicted that ST activated OAs would report more TRI than ST relief OAs and YAs. A one-way analysis of variance (ANOVA) for proportion of TRI revealed an effect of condition, $F(3,116)=5.201, p=.002, \eta^{2} .=.120$. ST activated OAs reported more TRI than YAs, $t(58)=-3.362, p=.001, d=.885$ and ST relief OAs, $t(58)=-2.694, p=.009, d=.608$. ST activated OAs also reported a numerically higher proportion of TRI than OA controls, $t(58)=-1.461, p=.149, d=.330$, although this difference was not statistically significant. The overall ANOVA including both TUTs and TRI confirmed a difference between type of mind-wandering, $F(1,116)=8.815, p=.004, \eta^{2}=.071$. An interaction of mind-wandering type with condition was also obtained, $F(3,116)=7.604, p<$ $.001, \eta^{2}=.164$, as younger adults reported less TRI relative to TUTs $(d=.6199)$ whereas each of the older adult conditions reported more TRI relative to TUTs (dcontrol $=.6854$, $d S T$ relief $=$ .4647 , dST activated $=-1.003 ; p s<.05$ ). There were no group differences in proportion of ontask thoughts, $F(3,116)=1.615, p=.190, \eta^{2}=.040$.

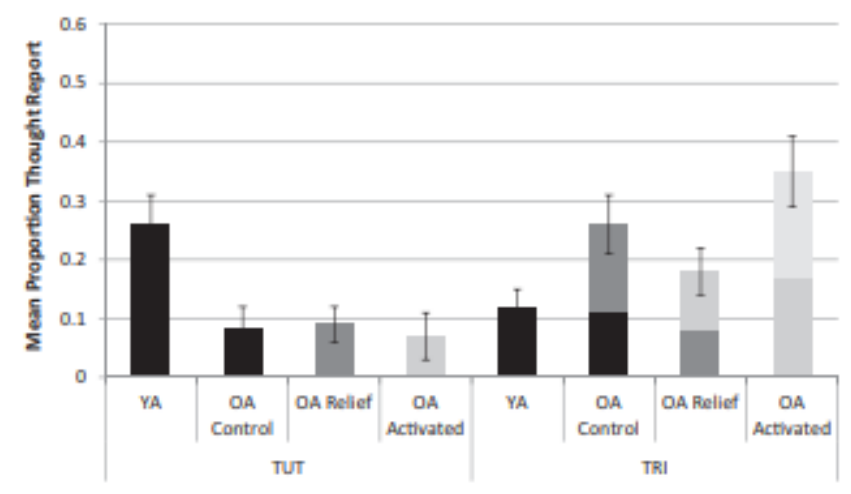

Figure 1. Mean proportions of probe-caught thought types during the operation task span. Bars indicate standard errors. Task-related interference (TRI) is broken down into proactive TRI (thinking about task strategy or approach) represented by the lighter portions of the TRI columns and reactive TRI (thinking about task performance) represented by the darker portion of the TRI columns. Standard error bars on the TRI columns represent the standard error for overall mean proportion of TRI. YA = younger adult; TUT $=$ task-unrelated thoughts; $\mathrm{OA}=$ older adults.

We made no predictions regarding which group would report the most proactive TRI. There was a significant effect of condition on proactive TRI, $F(3,116)=3.097, p=.030, \eta^{2}=.074$, with ST activated OAs reporting more proactive TRI than YAs, $t(58)=-2.312, p<.001, d=.635$. ST activated OAs also reported more proactive TRI than did ST relief OAs and control OAs, although these differences were not significant, $t(58)=-1.978, p=.053, d=.517$ and, $t(58)=$ $-1.336, p=.096, d=.345$, respectively. We did anticipate group differences in reactive TRI, in accordance with the anticipated influence on ST on worry-laden monitoring. A significant effect of condition for reactive TRI was found, $F(3,116)=2.702, p=.049, \eta^{2}=.065$, with ST activated OAs reporting more reactive TRI than YAs, $t(58)=-2.719, p=.009, d=.752$. ST activated OAs did not, however, report significantly more reactive TRI than ST relief OAs, $t(58)$ $=-1.611, p=.057, d=.414$ or control OAs, $t(58)=-0.528, p=.280, d=.137$. 
Within the OA sample, we predicted that ST activated OAs would have the lowest letter recall accuracy and ST relief OAs would have the highest letter recall accuracy. A main effect of condition was found, $F(3,116)=3.226, p=.025, \eta^{2}=.079$. As predicted, OAs in the ST activated condition had lower letter recall accuracy than YAs, $t(58)=2.704, p=.009, d=.690$, the ST relief OAs, $t(58)=2.391, p=.020, d=.613$, and control OAs, $t(58)=1.817, p=.075, d=$ .394. Because our ST manipulation focused on memory-related ST, and because there is not a wide-spread stereotype regarding age and mathematical ability, we made no predictions regarding the effects of condition on math verification accuracy within the OA sample. A main effect of condition was found for math verification accuracy, $F(3,116)=2.910, p=.038, \eta^{2}=$ .071 , with YAs obtaining higher math accuracy than did the ST activated OA group, $t(58)=$ 2.327, $p=.024, d=.698$ but not the ST relief OA $(d=.600)$ or control $(d=.447)$ OA groups $(p s$ $>$.05). Although the ST activated OA group numerically had the lowest math accuracy of the four groups, math accuracy did not differ significantly between control and ST relief $(d=.229)$, control and ST activated $(d=.405)$, and ST relief and activated $(d=.200)$ OAs $(p s>.05)$.

\section{Analysis of Supplementary Self-Report Measures}

In addition to the online thought probes embedded in the OSPAN task, we included additional posttask questions to gain a better understanding of which factors influence mind-wandering and task performance (see Table 1 ). We found an effect of condition on DSSQ TUT scores, $F(3,113)$ $=5.753, p=.001, \eta^{2}=.132$. The YA group, in addition to having the highest probe-caught TUTs, also had higher DSSQ TUT scores than control $(d=.728)$, ST relief $(d=.827)$, and ST activated $(d=.717)$ OAs ( $p$ s $<.05)$. Overall, DSSQ TUT scores were positively correlated with probe-caught TUT reports, $r=.416, p<.001$. We found no effect of condition on DSSQ TRI scores, $F(3,113)=1.646, p=.183, \eta^{2}=.042$, although DSSQ TRI scores were positively correlated with probe-caught TRI reports, $r=.347, p<.001$.

Because affect has been found to influence mind-wandering and because negative affect (Kane et al., 2007; Smallwood et al., 2009) we included the PANAS (Watson et al., 1988). Consistent with past findings, there were age differences in positive affect, $F(3,113)=12.035, p<.001$, $\eta^{2}=.242$, with the YA group reporting lower positive affect than control $(d=1.010)$, ST relief $(d=1.212)$ and ST activated $(d=1.076)$ OAs $(p s<.05)$. There was no effect of condition on negative affect, $F(3,113)=1.979, p=.121, \eta^{2}=.050$. ST activated OAs did not differ in affect compared with ST relief OAs, despite the fact that they engaged in more TRI. Several singleitem, Likert-scale posttask questions were included to measure factors that might influence mind-wandering and recall performance, along with the MIA (Dixon \& Hultsch, 1984). These measures along with correlations between mind-wandering variables, OSPAN performance, and posttask measures are discussed in the online supplemental materials.

\section{DISCUSSION}

The current study tested the CF $\times$ C framework of mind-wandering (McVay \& Kane, 2010) by manipulating current concerns about cognitive performance in OAs. We found that ST activated OAs reported significantly more TRI than did OAs relieved of ST. ST activated OAs also had memory performance that was significantly worse than the performance of ST relief OAs. These 
results replicate past work on ST in female YAs showing that activation of ST results in increased TRI and impaired math performance (Jordano \& Touron, 2015).

This finding that ST increases TRI supports the CF $\times$ C framework (McVay \& Kane, 2010), which proposes that frequency and content of mind-wandering is determined, in part, by the degree to which one's current concerns are activated by the environmental context. It is worth noting that control OAs who received no explicit ST activation had proportions of TRI and math verification accuracy that were intermediate between those of the ST activated OA group and the ST relief OA group. The difference in proportion of TRI between ST activated OAs and control OAs was only numerically different and not statistically significant, and it is possible that the normal laboratory testing environment is sufficient to trigger increased current concerns about cognition in OA research volunteers. This may explain the typical finding of activated TRI in OAs compared with YAs (Frank et al., 2015; Jackson \& Balota, 2012; Krawietz et al., 2012; McVay et al., 2012a, 2013).

Although increased proactive TRI might be expected to improve task performance, this was not the case in the current study. Although ST activated OAs indicated mind-wandering about task approach significantly more than both YAs and ST relief OAs, this group also had the worst performance. Overall, proportion of probe-caught TRI, proportion of probe-caught reactive TRI, and proportion of probe-caught proactive TRI, were all negatively correlated with OSPAN letter recall performance. It is possible that such strategizing does improve task performance in different tasks or in more extensive training situations; future research should explore these possibilities. It is also possible that participants were not considering potentially beneficial strategies to implement on future trials when they chose the proactive TRI response option on the online thought probes. Instead, participants may have been evaluating past or current strategies when they chose proactive TRI, which we would not necessary expect to lead to improved performance on future trials. More work is needed to determine the exact contents of participants' thoughts when they indicate TRI on online thought probes.

Our results may be explained by different accounts of ST. It is possible that increased TRI in stereotyped OAs resulted from cognitive resources being diverted away from the task and toward processing information associated with negative stereotypes (Schmader \& Johns, 2003), allowing mind-wandering experiences to enter into consciousness and disrupt performance. It is also possible that TRI did not result from diverted cognitive resources, and that OAs' prepotent tendency to engage in TRI was instead strengthened by ST (Jamieson \& Harkin2, 2007). More work is needed to determine the mechanism by which ST increases TRI. The current studies provide insight into why OAs traditionally report a higher mean proportion of TRI than do YAs, even though they report less mind-wandering overall (Frank et al., 2015; Giambra, 1989; Grodsky \& Giambra, 1990; Jackson \& Balota, 2012; Krawietz et al., 2012; McVay et al., 2012a, 2013). This research provides support for the CF $\times$ C framework (McVay \& Kane, 2010) of mind-wandering by demonstrating a role of personal concerns and environmental context on the frequency and content of mind-wandering. This framework can be further tested by activating current, everyday concerns in in both YAs and OAs, and examining whether this manipulation increases task-unrelated thought. 
In conclusion, the current study provides evidence for ST as a mind-wandering trigger. OAs relieved of ST exhibited decreased TRI and improved accuracy on a memory task compared with OAs with activated memory-related ST. Current concerns activated by instructional manipulations and the testing environment appear to be one cause for more frequent TRI in OAs.

\section{REFERENCES}

Barber, S. J., \& Mather, M. (2014). Stereotype threat in older adults: When and why does it occur, and who is most affected? In P.Verhaeghen \& C.Hertzog (Eds.), The Oxford handbook of emotion, social cognition, and problem solving during adulthood (pp. 302-320). New York, NY: Oxford University Press.

Beilock, S. L., Rydell, R. J., \& McConnell, A. R. (2007). Stereotype threat and working memory: Mechanisms, alleviation, and spillover. Journal of Experimental Psychology: General, 136, 256276. 10.1037/0096-3445.136.2.256

Cadinu, M., Maass, A., Rosabianca, A., \& Kiesner, J. (2005). Why do women underperform under stereotype threat? Evidence for the role of negative thinking. Psychological Science, 16, 572-578. 10.1111/j.0956-7976.2005.01577.x

Chasteen, A. L., Bhattacharyya, S., Horhota, M., Tam, R., \& Hasher, L. (2005). How feelings of stereotype threat influence older adults' memory performance. Experimental Aging Research, 31, 235-260. 10.1080/03610730590948177

Conway, A. R. A., Kane, M. J., Bunting, M. F., Hambrick, D. Z., Wilhelm, O., \& Engle, R. W. (2005). Working memory span tasks: A methodological review and user's guide. Psychonomic Bulletin \& Review, 12, 769-786. 10.3758/BF03196772

Dixon, R. A., \& Hultsch, D. F. (1984). The Metamemory in Adulthood (MIA) instrument. Psychological Documents, 14, 3.

Ekstrom, R. B., French, J. W., \& Harman, H. H. (1976). Kit of factor-referenced cognitive tests. Princeton, NJ: Education Testing Service.

Ennis, G. E., Hess, T. M., \& Smith, B. T. (2013). The impact of age and motivation on cognitive effort: Implications for cognitive engagement in older adulthood. Psychology and Aging, 28, 495504. 10.1037/a0031255

Frank, D. J., Nara, B., Zavagnin, M., Touron, D. R., \& Kane, M. J. (2015). Validating older adults’ reports of less mind-wandering: An examination of eye movements and dispositional influences. Psychology and Aging, 30, 266-278. 10.1037/pag0000031

Giambra, L. M. (1989). Task-unrelated-thought frequency as a function of age: A laboratory study. Psychology and Aging, 4, 136-143. 10.1037/0882-7974.4.2.136 
Grodsky, A., \& Giambra, L. M. (1990). The consistency across vigilance and reading tasks of individual differences in the occurrence of task-unrelated and task-related images and thoughts. Imagination, Cognition and Personality, 10, 39-52. 10.2190/6QG5-CXVV-4XUR7P3K

Hasher, L., \& Zacks, R. T. (1988). Working memory, comprehension, and aging: A review and a new view. In G. H.Bower (Ed.), The psychology of learning and motivation (Vol. 22, pp. 193225). New York, NY: Academic Press. 10.1016/S0079-7421(08)60041-9

Hertzog, C., \& Hultsch, D. F. (2000). Metacognition in adulthood and old age. In F. I. M.Craik \& T. A.Salthouse (Eds.), The handbook of aging and cognition (pp. 417-466). Mahwah, NJ: Erlbaum.

Hess, T. M., Auman, C., Colcombe, S. J., \& Rahhal, T. A. (2003). The impact of stereotype threat on age differences in memory performance. The Journals of Gerontology: Psychological Sciences and Social Sciences, 58(1), P3-P11. 10.1093/geronb/58.1.P3

Hummert, M. L. (1999). A social cognitive perspective on age stereotypes. In T. M.Hess \& F.Blanchard-Fields (Eds.), Social cognition and aging (pp. 175-196). New York, NY: Academic Press. 10.1016/B978-012345260-3/50009-4

Jackson, J. D., \& Balota, D. A. (2012). Mind-wandering in younger and older adults: Converging evidence from the Sustained Attention to Response Task and reading for comprehension. Psychology and Aging, 27, 106-119. 10.1037/a0023933

Jamieson, J. P., \& Harkins, S. G. (2007). Mere effort and stereotype threat performance effects. Journal of Personality and Social Psychology, 93, 544-564. 10.1037/0022-3514.93.4.544

Jordano, M. J., \& Touron, D. R. (2015, November). Mind-wandering and the Role of Stereotype Threat. Poster session presented at the 56th Annual Convention of the Psychonomic Society, Chicago, IL.

Kane, M. J., Brown, L. H., McVay, J. C., Silvia, P. J., Myin-Germeys, I., \& Kwapil, T. R. (2007). For whom the mind wanders, and when: An experience-sampling study of working memory and executive control in daily life. Psychological Science, 18, 614-621. 10.1111/j.14679280.2007.01948.x

Killingsworth, M. A., \& Gilbert, D. T. (2010). A wandering mind is an unhappy mind. Science, 330, 932. 10.1126/science.1192439

Kopp, K., D’Mello, S., \& Mills, C. (2015). Influencing the occurrence of mind wandering while reading. Consciousness and Cognition, 34, 52-62. 10.1016/j.concog.2015.03.003

Krawietz, S. A., Tamplin, A. K., \& Radvansky, G. A. (2012). Aging and mind wandering during text comprehension. Psychology and Aging, 27, 951-958. 10.1037/a0028831 
Masicampo, E. J., \& Baumeister, R. F. (2011). Consider it done! Plan making can eliminate the cognitive effects of unfulfilled goals. Journal of Personality and Social Psychology, 101, 667683. 10.1037/a0024192

Matthews, G., Joyner, L., Gilliland, K., Huggins, J., \& Falconer, S. (1999). Validation of a comprehensive stress state questionnaire: Towards a state big three? In I.Merville, I. J.Deary, F.DeFruyt, \& F.Ostendorf (Eds.), Personality psychology in Europe (pp. 335-350). Tilburg, the Netherlands: Tilburg University Press.

McVay, J. C., \& Kane, M. J. (2009). Conducting the train of thought: Working memory capacity, goal neglect, and mind wandering in an executive-control task. Journal of Experimental Psychology: Learning, Memory, and Cognition, 35, 196-204. 10.1037/a0014104

McVay, J. C., \& Kane, M. J. (2010). Does mind wandering reflect executive function or executive failure? Comment on Smallwood and Schooler (2006). and Watkins (2008). Psychological Bulletin, 136, 188-197. 10.1037/a0018298

McVay, J. C., \& Kane, M. J. (2012a). Why does working memory capacity predict variation in reading comprehension? On the influence of mind wandering and executive attention. Journal of Experimental Psychology: General, 141, 302-320. 10.1037/a0025250

McVay, J. C., \& Kane, M. J. (2012b). Drifting from slow to “D’oh!”: Working memory capacity and mind wandering predict extreme reaction times and executive control errors. Journal of Experimental Psychology: Learning, Memory, and Cognition, 38, 525-549. 10.1037/a0025896

McVay, J. C., \& Kane, M. J. (2013). Dispatching the wandering mind? Toward a laboratory method for cuing "spontaneous" off-task thought. Frontiers in Psychology, 4, 570. 10.3389/fpsyg.2013.00570

McVay, J. C., Meier, M. E., Touron, D. R., \& Kane, M. J. (2013). Aging ebbs the flow of thought: Adult age differences in mind wandering, executive control, and self-evaluation. Acta Psychologica, 142, 136-147. 10.1016/j.actpsy.2012.11.006

Mrazek, M. D., Chin, J. M., Schmader, T., Hartson, K. A., Smallwood, J., \& Schooler, J. W. (2011). Threatened to distraction: Mind wandering as a consequence of stereotype threat. Journal of Experimental Social Psychology, 47, 1243-1248. 10.1016/j.jesp.2011.05.011

Mrazek, M. D., Franklin, M. S., Phillips, D. T., Baird, B., \& Schooler, J. W. (2013). Mindfulness training improves working memory capacity and GRE performance while reducing mind wandering. Psychological Science, 24, 776-781. 10.1177/0956797612459659

Rummel, J., \& Boywitt, C. D. (2014). Controlling the stream of thought: Working memory capacity predicts adjustment of mind-wandering to situational demands. Psychonomic Bulletin \& Review, 21, 1309-1315. 10.3758/s13423-013-0580-3

Salthouse, T. A. (1991). Mediation of adult age differences in cognition by reductions in working memory and speed of processing. Psychological Science, 2, 179-183. 10.1111/j.14679280.1991.tb00127.x 
Salthouse, T. A. (1993). Speed mediation of adult age differences in cognition. Developmental Psychology, 29, 722-738. 10.1037/0012-1649.29.4.722

Schaie, K. W. (1994). The course of adult intellectual development. American Psychologist, 49, 304-313. 10.1037/0003-066X.49.4.304

Schmader, T., Forbes, C. E., Zhang, S., \& Mendes, W. B. (2009). A metacognitive perspective on the cognitive deficits experienced in intellectually threatening environments. Personality and Social Psychology Bulletin, 35, 584-596. 10.1177/0146167208330450

Schmader, T., \& Johns, M. (2003). Converging evidence that stereotype threat reduced working memory capacity. Journal of Personality and Social Psychology, 85, 440-452. 10.1037/00223514.85.3.440

Schmader, T., Johns, M., \& Forbes, C. (2008). An integrated process model of stereotype threat effects on performance. Psychological Review, 115, 336-356. 10.1037/0033-295X.115.2.336

Schuster, C., Martiny, S. E., \& Schmader, T. (2015). Distracted by the unthought-Suppression and reappraisal of mind wandering under stereotype threat. PLoS ONE, 10, e0122207. 10.1371/journal.pone.0122207

Seli, P., Carriere, J. S. A., Levene, M., \& Smilek, D. (2013). How few and far between? Examining the effects of probe rate on self-reported mind wandering. Frontiers in Psychology, 4, 430. 10.3389/fpsyg.2013.00430

Smallwood, J., Fitzgerald, A., Miles, L. K., \& Phillips, L. H. (2009). Shifting moods, wandering minds: Negative moods lead the mind to wander. Emotion, 9, 271-276. 10.1037/a0014855

Smallwood, J., McSpadden, M., \& Schooler, J. W. (2008). When attention matters: The curious incident of the wandering mind. Memory \& Cognition, 36, 1144-1150. 10.3758/MC.36.6.1144

Steele, C. M., \& Aronson, J. (1995). Stereotype threat and the intellectual test performance of African Americans. Journal of Personality and Social Psychology, 69, 797-811. 10.1037/00223514.69.5.797

Unsworth, N., \& McMillan, B. D. (2013). Mind wandering and reading comprehension: Examining the roles of working memory capacity, interest, motivation, and topic experience. Journal of Experimental Psychology: Learning, Memory, and Cognition, 39, 832842. 10.1037/a0029669

Watson, D., Clark, L. A., \& Tellegen, A. (1988). Development and validation of brief measures of positive and negative affect: The PANAS scales. Journal of Personality and Social Psychology, 54, 1063-1070. 10.1037/0022-3514.54.6.1063 